\title{
Ultraviolet Box: An Innovative In-house Use of Ultraviolet Irradiation for Conservation of Respirators in COVID-19 Pandemic
}

\author{
Prashant Kumar ${ }^{1}$, Dhruva Chaudhry ${ }^{2}$, Aparna Parmar ${ }^{3}$, Diksha Tyagi ${ }^{4}$, Manjunath BG ${ }^{5}$, Pawan Kumar Singh ${ }^{6}$
}

\begin{abstract}
Aim: To use ultraviolet (UV) radiations in an indigenous method for sterilization of respirators for reuse during COVID-19 outbreak.

Background: COVID-19 outbreak has infected more than 200 countries. In India, till now, more than 100,000 cases have been reported. Healthcare workers are at high risk of developing infections being in the frontline of taking care of COVDI-19 cases. The demands of personal protective equipment (PPE) are increasing, but the same is not matched with supply due to various reasons. In such scenarios, reusing respirators and face shields is an alternative. UV radiations have quick action and are able to preserve the quality of respirators. We have developed a UV box for surface sterilization of respirators with an intention to reuse.

Technique: A thermocol box was taken from the central drug store and was fitted with two UV tubes of $254 \mathrm{~nm}$ wavelength procured from local service center of water purifiers. The position of the two tubes was such that one was near the base while other was fixed at the top. An aluminum mesh frame was placed in the middle of the box to act as a platform. The roof of the box was converted into a lid. The effectiveness of assembly was tested using culture of Escherichia coli and Staphylococcus aureus. In addition, a biological indicator tube containing test strip with spores of Bacillus atrophaeus was also exposed to UV light for a predefined duration, which did not show any color change after incubation for 48 hours. Conclusion: Our prototype assembly with supported efficacy from microbiological tests is an option for use of UV light within available resources for disinfection and reuse of scarce supplies of personal protective equipment.

Clinical significance: UV box can help in meeting the demand supply deficit for respirators, face shields, and goggles that are paramount for the protection of HCW.

Keywords: COVID 19, N95 respirators, SARS-COV-2, UV radiation.

Indian Journal of Critical Care Medicine (2020): 10.5005/jp-journals-10071-23534
\end{abstract}

\section{BACKGROUND}

COVID-19 outbreak has been recently declared by the World Health Organization (WHO) as pandemic. Per situation reports of $\mathrm{WHO}$, the COVID-19 outbreak has involved over 200 countries. ${ }^{1}$ In India, more than 100,000 individuals have been infected, and over 3,000 deaths have been reported until the writing of this article. ${ }^{2}$ Healthcare workers $(\mathrm{HCW})$ are at particularly high risk of being infected, as they are actively involved in caring for these individuals. ${ }^{3}$ In such scenarios, personal protective equipment (PPE), such as cover-all, respirators, and face shield, has become vital for HCWs to continue serving COVID19 cases. Due to the rise in number of cases, there has been a huge demand for these items. Over time multiple international agencies such as Center for Disease Control and Prevention have released guidelines for its judicious use and potential upcoming scarcity. ${ }^{4}$

Most of these equipment are for single-time use only, but it is anticipated that in upcoming time there will be a shortage of these items. Reuse after sterilizations has been proposed, particularly for respirators and face shields. In this current pandemic, the dearth of vital PPE can have catastrophic consequences.

Ultraviolet (UV) radiation is a form of nonionizing electromagnetic radiation. According to the wavelength, in the spectrum, UV radiation come between visible light and $\mathrm{X}$-ray. UV radiation is classified based on its effect on living tissue and wavelength. It is commonly divided into three major groups: UV-A (320-400 nm), UV-B (290-320), and UV-C (200-290). 5,6

Ultraviolet irradiation has an ability to destroy microorganisms by causing instability in double bonds of carbon atoms in nucleic
${ }^{1}$ Department of Anaesthesiology and Critical Care, Pt Bhagwat Dayal Sharma Post Graduate Institute of Medical Sciences, Rohtak, Haryana, India

2,4-6 Department of Pulmonary and Critical Care Medicine, Pt Bhagwat Dayal Sharma Post Graduate Institute of Medical Sciences, Rohtak, Haryana, India

${ }^{3}$ Department of Microbiology, Pt Bhagwat Dayal Sharma Post Graduate Institute of Medical Sciences, Rohtak, Haryana, India

Corresponding Author: Pawan Kumar Singh, Department of Pulmonary and Critical Care Medicine, Pt Bhagwat Dayal Sharma Post Graduate Institute of Medical Sciences, Rohtak, Haryana, India, Phone: +91 8437013094, e-mail: ga.ps.complete@gmail.com

How to cite this article: Kumar P, Chaudhry D, Parmar A, Tyagi D, BG Manjunath, Singh PK. Ultraviolet Box: An Innovative In-house Use of Ultraviolet Irradiation for Conservation of Respirators in COVID-19 Pandemic. Indian J Crit Care Med 2020;24(8):713-715.

Source of support: Nil

Conflict of interest: None

acids. UV radiation, particularly in the wavelength between 200 and $300 \mathrm{~nm}$, has been studied and found to be effective as germicidal. The peak of the effectivity is around $260 \mathrm{~nm}$, which has the highest absorption by bacterial DNA. ${ }^{7}$

For the process of sterilization, UV radiation has been commonly used, especially for airborne infections like tuberculosis and influenza. ${ }^{8,9}$ Closed UV cabinets have come up for the purpose of sterilization. The important advantages of such cabinets are UV 
irradiation from opposite ends, portability, and compact size. Such cabinets are commercially available for the surface sterilization, but given the high cost and administrative measures of lockdown and cessation of transport, even their procurement is an uphill task. The average cost of such units is above INR 25,000 .

The effectiveness of UV cabinet for the purpose of sterilization has been evaluated, in a bench-side study, by Moore et al., and they concluded that it can provide rapid as well as effective disinfection of patient-related items. They used bacterial spores as well as viruses to establish the efficacy of the same. ${ }^{10}$

In a study based on similar concept, Cadnum et al. assessed the efficacy of UV cabinet to sterilize and reuse components of PPE during COVID-19 pandemic. They concluded that a high-level disinfection of $\mathrm{N} 95$ respirators was effective and feasible. ${ }^{11}$

On a similar note, we here describe an indigenous method of making UV box. We have used this box, after testing for its efficacy, for sterilization of respirators, face shields, and goggles.

Following the essential microbiological testing and administrative approval, this UV box was used at high-intensity COVID-19 dedicated areas of the hospital

\section{TECHNIQUe}

A thermocol box of size $24 \times 24 \times 20$ inches was taken from the central drug store (vaccines/drugs were delivered in it). An aluminum mesh frame was placed in the middle of the box to act as a platform (Fig. 1A). The box was fitted with two 11 watts UV tubes of $254 \mathrm{~nm}$ wavelength procured from local service center of commercially used water purifiers. The position of the two tubes was such that one was near the base, while the other was fixed at the top (on the roof of the box) (Fig. 1B). A clear plastic bag, containing the $\mathrm{N} 95$ respirator, was placed over the mesh frame in such a way that both of its surfaces are being exposed to UV radiations (Fig. 1B). The roof of the box was converted into lid, and seal was developed by modifying the edges. An airtight seal was not aimed, as it was not necessary. Silver/aluminum paper/foil coating was done over the box so as to protect the operator from any accidental exposure (Fig. 1C). The entire assembly costed less than INR 1,000, as most of the items used were arranged from scrap material.

The effectiveness of assembly was tested using culture of Escherichia coli and Staphylococcus aureus. Overnight growth of bacteria was harvested in normal saline, and opacity was adjusted to 0.5 McFarland Standard. Ten microliter suspension of each was spread over nutrient agar plates, which were then exposed to UV light for a period of 30 minutes, 1 hour, and 2 hours duration. The bacterial suspensions were subjected to viable count using 10 log dilutions to confirm the count. Reduction in the viable count was expressed as ratio of initial viable count to viable count after exposure to UV light. The results suggested approximately four log reduction in viable count after exposure to UV light for 30 minutes. No bacterial growth was seen after exposure of 1 hour and 2 hours (Fig. 2).

In addition, a biological indicator tube containing test strip with spores of Bacillus atrophaeus was also exposed to UV light for a duration of 2 hours which did not show any color change after incubation for 48 hours, hence strengthening our belief that the box has enough UV energy to make the surface sterile. Disinfected masks had their structures maintained after the procedure. Also, it did not affect the functional aspect, as there was no increase in resistance as felt subjectively.

For its final use, in one sitting, 10 N95 respirators or 5 goggles or 2 face shields could be sterilized in one sitting. Each cycle was run for 30 minutes, and after 6 such cycles, a break of 1 hour was provided to the device. The aluminum/silver foil was not changed till there was physical soiling of the surface. For physically soiled inside, dry mopping was used with a cloth, and this was followed by empty run for one cycle. The most important precaution used by the operator was to switch off the box before opening the lid.

\section{Discussion}

Shortage of respirators and face shields were foreseen in our institute. There were two main factors behind it: first the rise in demand and second its use. This was expected with the rise in number of cases and recruitment of human resources at various sites. So the number used per day was rising exponentially. Second, transport restriction and lockdown imposed by the administrative authorities led to unavailability of the items. This led us to search for various methods of reuse of respirators and face shields. In the literature, various methods of disinfection has been described by autoclaving and ethylene trioxide. ${ }^{12,13}$ But all these methods had two major drawbacks; one was, post-sterilization the physical quality of mask was showing deterioration, and there was an increase in resistance to exhaled breath. The second drawback was the turnaround time of these machines, which were also catering to the needs of various other departments in the hospital.

UV radiation was seen as an answer to both of our problems, but commercially available boxes were difficult to procure, and the cost was prohibitive. This was followed by the idea of developing a UV box in house. In a study by Lindsley et al., UV germicidal irradiation was effective in sterilizing the $\mathrm{N} 95$ respirators as well as preserving the quality of $\mathrm{N} 95$ respirators in the form of particle penetration and flow resistance. ${ }^{14}$ Similar results were also demonstrated in a study conducted by Mills et al. ${ }^{15}$
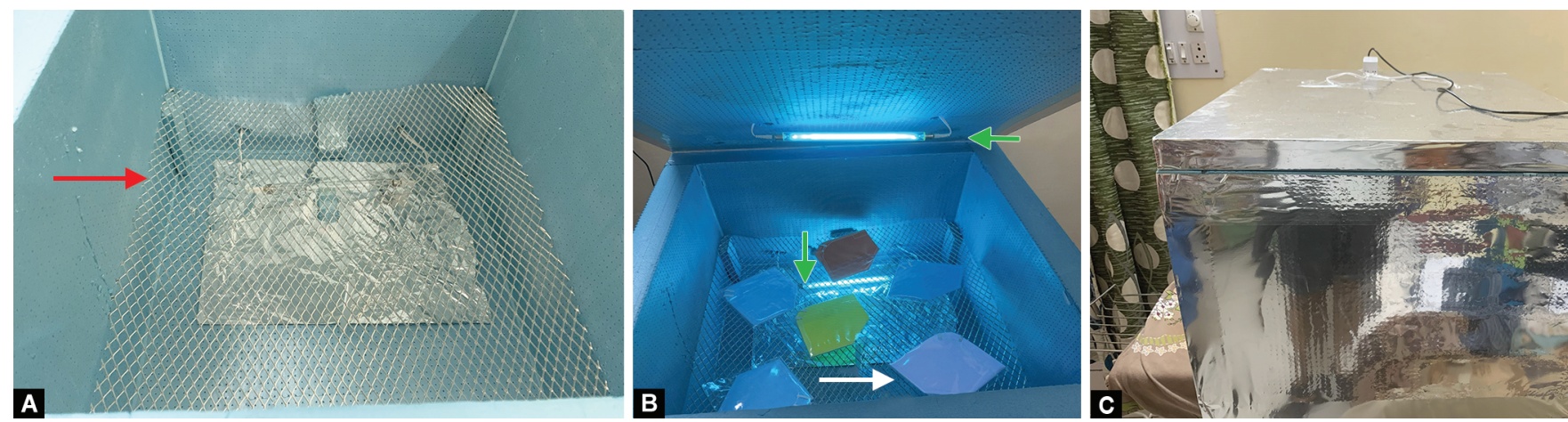

Figs 1 A to C: Ultraviolet (UV) box assembly: (A) Aluminum mesh placed in the middle of the box (red arrow); (B) Switched on UV tubes, one in the lid and one at the base (green arrows) along with respirators lying on the aluminum mesh (white arrow); (C) Outer view of the box showing the silver foil cover 


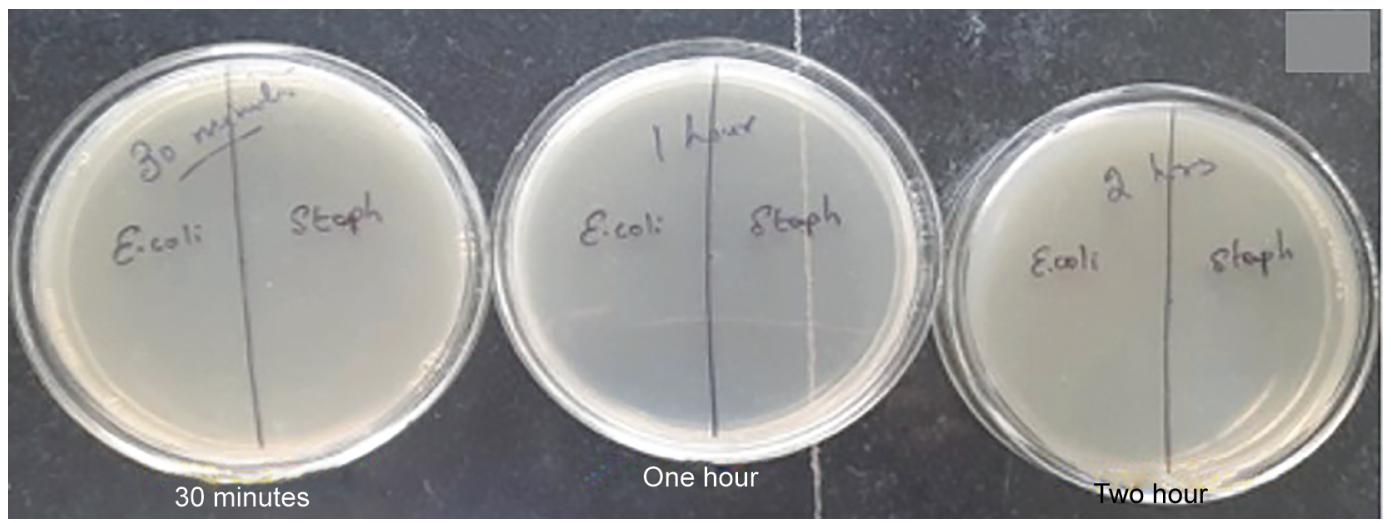

Fig. 2: Culture plates in series showing the growth of Escherichia coli and Staphylococcus aureus at 30 minutes, 1 hour, and 2 hours

Exposure to water, soap, alcohol, and hypochlorite causes a decrease in transparency of face shields and goggles. This leads to haziness and poor vision for the HCW. Using UV radiations for the same was also helpful in finding an answer to this problem.

All the materials used in the making of this box are commonly available and are significantly cheap. We propose that common use of these boxes at the sites, where there is high number of respirators/face shields being disposed-off, can lead to safe reuse and decrease in the biomedical waste that is currently being generated.

There were a few drawbacks with the study and the design. First, the aluminum mesh placed in the middle of the box could hold only lighter items. It is likely that in future with its extensive use, there might be a need to replace the mesh. In addition to this, we could not test of virucidal efficiency of the box, but with the similar technique, virucidal activity of the UV cabinet was demonstrated by Cadnum et al. against adenovirus. ${ }^{11}$ Finally, we used only subjective method to assess the quality of respirators, and no objective methods were used. Although the limitation was due to unavailability of testing facility, we feel that for real-world applicability, subjective proof of preserved quality should suffice.

\section{Conclusion}

To conclude, the judicious and careful use of UV light can help disinfect the precious and scarce resources of PPE in global pandemic. Our prototype assembly with supported efficacy from microbiological tests is an option for use of UV light within available resources for disinfection and reuse of scarce resources.

\section{Clinical Significance}

- UV box can help in meeting the demand-supply deficit for respirators, face shields, and goggles, which are among the PPE of HCW with paramount importance

- UV box can also add to the armamentarium of the hospitals to deal with particular biomedical waste which is being generated at enormous amounts.

\section{References}

1. Organization WH. Coronavirus disease (COVID-19) Situation Report120 2020. Available from: https://www.who.int/docs/default-source/ coronaviruse/situation-reports/20200519-covid-19-sitrep-120. pdf?sfvrsn=515cabfb_2.
2. Welfare MoHaF. COVID-19 INDIA India: Government of India; 2020. Available from: https://www.mohfw.gov.in/.

3. Wilson NM, Norton A, Young FP, Collins DW. Airborne transmission of severe acute respiratory syndrome coronavirus-2 to Healthcare workers: a narrative review. Anaesthesia 2020;75(8):1086-1095. DOI: 10.1111/anae.15093.

4. Prevention CfDCa. Strategies for Optimizing the Supply of N95 Respirators USA: U.S. Department of Health and Human Services; 2020. Available from: https://www.cdc.gov/coronavirus/2019-ncov/ hcp/respirators-strategy/index.html.

5. Prevention CfDCa. UV Radiation 2019. Available from: https://www. cdc.gov/nceh/features/uv-radiation-safety/index.html.

6. Diffey BL. Sources and measurement of ultraviolet radiation. Methods 2002;28(1):4-13. DOI: 10.1016/S1046-2023(02)00204-9.

7. Cutler TD, Zimmerman JJ. Ultraviolet irradiation and the mechanisms underlying its inactivation of infectious agents. Anim Health Res Rev 2011;12(1):15-23. DOI: 10.1017/S1466252311000016.

8. Walker CM, Ko G. Effect of ultraviolet germicidal irradiation on viral aerosols. Environ Sci Technology 2007;41(15):5460-5465. DOI: 10.1021/es070056u.

9. Welch D, Buonanno M, Grilj V, Shuryak I, Crickmore C, Bigelow AW, et al. Far-UVC light: a new tool to control the spread of airbornemediated microbial diseases. Sci Rep 2018;8(1):2752. DOI: 10.1038/ s41598-018-21058-w.

10. Moore G, Ali S, Cloutman-Green EA, Bradley CR, Wilkinson MA, Hartley $J C$, et al. Use of UV-C radiation to disinfect non-critical patient care items: a laboratory assessment of the nanoclave cabinet. BMC Inf Dis 2012;12:174. DOI: 10.1186/1471-2334-12-174.

11. Cadnum JL, Li DF, Redmond SN, John AR, Pearlmutter B, Donskey CJ. Effectiveness of ultraviolet- $C$ light and a high-level disinfection cabinet for decontamination of N95 respirators. Pathog Immun 2020;5(1):52-67. DOI: 10.20411/pai.v5i1.372.

12. Viscusi DJ, Bergman MS, Eimer BC, Shaffer RE. Evaluation of five decontamination methods for filtering facepiece respirators. Ann Occup Hyg 2009;53(8):815-827.

13. Lore MB, Heimbuch BK, Brown TL, Wander JD, Hinrichs SH. Effectiveness of three decontamination treatments against influenza virus applied to filtering facepiece respirators. Ann Occup Hyg 2012;56(1):92-101.

14. Lindsley WG, Martin Jr SB, Thewlis RE, Sarkisian K, Nwoko JO, Mead $\mathrm{KR}$, et al. Effects of ultraviolet germicidal irradiation (UVGI) on N95 respirator filtration performance and structural integrity. J Occup Environ Hyg 2015;12(8):509-517. DOI: 10.1080/15459624.2015. 1018518.

15. Mills D, Harnish DA, Lawrence C, Sandoval-Powers M, Heimbuch BK. Ultraviolet germicidal irradiation of influenza-contaminated N95 filtering facepiece respirators. Am J Infect Control 2018;46(7):e49-e55. DOI: 10.1016/j.ajic.2018.02.018. 